\title{
Chronique des travaux universitaires soutenus en
} 2021

Jean-Pierre Watté

\section{OpenEdition}

\section{Journals}

Édition électronique

URL : https://journals.openedition.org/rao/7564

DOI : $10.4000 /$ rao. 7564

ISSN : $1775-3732$

Éditeur

Presses universitaires de Rennes

\section{Édition imprimée}

Date de publication : 21 décembre 2021

Pagination : 367-369

ISBN : 978-2-7535-8668-0

ISSN : 0767-709X

\section{Référence électronique}

Jean-Pierre Watté, "Chronique des travaux universitaires soutenus en 2021 », Revue archéologique de l'Ouest [En ligne], 37 | 2021, mis en ligne le 21 décembre 2021, consulté le 11 octobre 2022. URL:

http://journals.openedition.org/rao/7564; DOI : https://doi.org/10.4000/rao.7564 


\section{Chronique des travaux universitaires soutenus en 2021}

JEAN-PIERRE WATtÉ

Nous continuons ici la recension des thèses et mémoires d'archéologie ou de disciplines connexes soutenus dans l'Ouest ainsi que celle des travaux concernant à un titre ou à un autre l'archéologie du Grand Ouest français, comme dans les précédents volumes. Compte tenu de la difficulté matérielle à obtenir un dépouillement exhaustif en la matière, la rédaction de la Revue archéologique de l'Ouest remercie chaleureusement par avance celles et ceux (notamment les lauréats et leurs directeurs de recherche) qui voudront bien lui faire part des références susceptibles de figurer utilement dans cette chronique, quel que soit le cadre - français ou étranger - dans lequel ces travaux auront été conduits et, par là même, contribueront à les faire connaître.

\section{UNIVERSITÉ DE RENNES 1}

\section{Mémoires de master 1}

Charretier Pierre (2021), Étude palynologique du nord de la vallée du Blavet (56), sous la direction de Chantal Leroyer (ministère de la Culture, UMR 6566) et David Aoustin (CNRS, UMR 6566).

Herpin Charlotte (2021), Les restes végétaux en contexte funéraire durant le second âge du Fer et le Haut-Empire en Gaule-Apports des sources antiques, archéologiques et archéobotaniques, sous la direction de David Aoustin (CNRS, UMR 6566), Fabien Colleoni (université Rennes 2, UMR 6566) et Chantal Leroyer (ministère de la Culture, UMR 6566).

\section{Mémoire de master 2}

Rossignol Cédric (2020), Étude palynologique du site de Plougasnou/Saint-Jean-du-Doigt (Finistère), sous la direction de Chantal Leroyer (ministère de la Culture, UMR 6566) et David Aoustin (CNRS, UMR 6566).

\section{Thèse de doctorat}

Duval Hervé (2020), Les sites fortifiés littoraux et insulaires de la façade Manche-Atlantique de l'Europe : territoires, échanges et pouvoirs au Ier millénaire av. $n$. è., sous la direction de Marie-Yvane Daire (université Rennes 1).

\section{UNIVERSITÉ DE RENNES 2}

\section{Mémoires de master 1}

Hardy Audrey (2021), Les hommes et leurs navires durant l'âge du Bronze dans la Mer du Nord et la Manche, sous la direction de Cyril Marcigny (Inrap).

Le Diagon Mathieu (2021), Dynamique d'implantation des premiers peuplements de la vallée du Blavet (Morbihan-56) du Paléolithique final au Néolithique. Approche géoarchéologique, sous la direction de Yoann Chantreau (ministère de la Culture, UMR 6566).

\section{Mémoires de master 2}

BéLIer Benoît (2021), Du manoir à l'exploitation agricole. Étude du site des Alignés (Laval, Mayenne), de la fin du Moyen Âge à nos jours, sous la direction de Samuel Chollet et Pierre-Yves Laffont (université Rennes 2).

Levéziel Thomas (2021), Terroirs et habitat rural au Moyen Âge en Haute Bretagne. L'exemple de Trans-laForêt (Ille-et-Vilaine), sous la direction de Pierre-Yves Laffont et Magali Watteaux (université Rennes 2).

Mouchel dit Muscardin Claire (2021), Analyse spatiale et modélisation des systèmes de peuplement. Approche relationnelle des modes d'habitats et des structures d'occupation du sol en Normandie à l'âge du Bronze, sous la direction de Cyril Marcigny (Inrap).

\section{Thèses de doctorat}

Le Goff Laura (2020), Collecter, consommer, (ré)utiliser. L'exploitation des invertébrés marins entre Manche et Garonne au Moyen Âge et à l'Époque moderne, sous la direction de Pierre-Yves Laffont (université Rennes 2).

Tirel Mélissa (2020), De jeunes morts chez les vivants. Les pratiques funéraires destinées aux enfants en bas âge découverts hors des nécropoles de Gaule romaine, sous la direction de Fabien Colleoni et Mario Denti (université Rennes 2). 


\section{UNIVERSITÉ DE NANTES}

\section{Mémoires de master 1}

Allemand Charlotte (2018), Les thermes privés ruraux sur le territoire des Cénomans (Sarthe), sous la direction de Martial Monteil (université de Nantes).

Bellanger Mathilde (2019), Le temple à plan centré de la Vieille Vigne au Fief-Sauvin (Maine-et-Loire). Reprise des données de fouille, sous la direction de Martial Monteil (université de Nantes) et Gérard Aubin (ministère de la Culture).

Besson Émeric (2021), Premier inventaire du Mur de l'Atlantique à La Rochelle : la ligne de défense allemande du littoral rochelais, sous la direction de Gaëlle Dieulefet (université de Nantes).

Branchereau Simon (2020), La ville d'Auray (Morbihan) du XI au XVI siècle : état de la documentation et première approche morphologique, sous la direction de Julien Bachelier et Yves Henigfeld (université de Nantes).

Colin Matéo (2019). Les modes de défense et d'attaque des villes fortifiées de l'Antiquité tardive. Approche bibliographique, sous la direction de Martial Monteil (université de Nantes).

Criquetot Ophélie (2020), Les scramasaxes du musée Dobrée à Nantes, sous la direction de Yves Henigfeld et Édith Peytremann (université de Nantes).

Fichou-Martin Erwan (2019), Trottoirs et portiques dans la ville romaine de Rennes. Évolution et types d'aménagements, sous la direction de Martial Monteil (université de Nantes) et Gaëtan Le Cloirec (Inrap).

Grosbois Ombline (2019), Le mobilier céramique du comblement de la cave 3361 du quartier antique de la clinique Saint-Louis, sous la direction de Martial Monteil (université de Nantes) et Maxime Mortreau (Inrap).

Herbert Coline (2018), Les sépultures rupestres de l'Antiquité tardive et du haut Moyen Âge de l'Orbière à Saint-Georges-des-Sept-Voies (Maine-et-Loire), sous la direction de Martial Monteil (université de Nantes) et Lauré Déodat (CNRS, UMR 6566 CReAAH).

Le Du Félix (2021), Inventaire critique des hallebardes $d u$ Chalcolithique et du Bronze ancien en France et dans les îles Anglo-Normandes, sous la direction de Sylvie Boulud-Gazo (université de Nantes).

Lourdais Angel (2021), Les vestiges de la Seconde Guerre mondiale à Nantes (44), sous la direction de Gaëlle Dieulefet (université de Nantes).

Morgand Valentine (2021), La guimbarde du XIII au XVIII s. dans le Nord-Ouest de la France (Pays de la Loire, Bretagne, Normandie), sous la direction de
Yves Henigfeld (université de Nantes) et Jean Soulat, (LandArc).

Muzelet-Guedon Cloé (2021), Les carreaux de pavement de la celle grandmontaine de la Haye-aux-Bonshommes d'Avrillé (XII'-XVIII' s. - Maine-et-Loire), sous la direction de Yves Henigfeld (université de Nantes) et JeanFrançois Nauleau (Inrap).

Nemes Cassandra (2018), Dynamiques d'occupation du pays guérandais à l'époque romaine. Synthèse bibliographique et cartographique, sous la direction de Martial Monteil (université de Nantes).

Orieux Paul (2021), L'armement de l'âge du Fer en Vendée, sous la direction de Martial Monteil (université de Nantes) et Olivier Nillesse (Inrap).

Rivoallan Mathilde (2021), Équipement et armements des soldats français de la guerre 1914-1918, sous la direction de Gaëlle Dieulefet (université de Nantes).

Sannier Gwendoline (2021), Les épaves anglaises $d u$ XVIII $s$. de la façade atlantique. Le cas $d u$ HMS Maidstone (1747), sous la direction de Gaëlle Dieulefet (université de Nantes).

Semelin Swann (2020), Étude du verre antique du site de Saint-Lupien à Rezé (Loire-Atlantique), sous la direction de Martial Monteil (université de Nantes) et Stéphanie Raux (Inrap).

WaLter Valentin (2020), Une approche systémique de l'estuaire de la Vilaine à l'époque romaine (Ir s. av. J.-C. - V's. apr. J.-C.), sous la direction de Martial Monteil (université de Nantes).

\section{Mémoires de master 2}

Bellanger Mathilde (2020), L'édifice dit le "nymphée " de Gennes (Gennes-Val-de-Loire) : reprise des données du $X I X^{*}$ s. et observations contemporaines, sous la direction de Martial Monteil (université de Nantes).

Dupont Pauline (2021), Le remploi du bois d'épave et des pierres de lest dans l'architecture en baie de Bourgneuf à l'époque médiévale et moderne, sous la direction de Gaëlle Dieulefet et David Plouviez (université de Nantes).

Fichou-Martin Erwan (2020), Les espaces transitoires des chefs-lieux gallo-romains de la péninsule armoricaine : aménagements, évolutions et usages des bordures de rues de Vorgium, Fanum Martis et Darioritum, sous la direction de Martial Monteil (université de Nantes) et Gaëtan Le Cloirec (Inrap).

Grosвors Ombline (2020), La céramique antique d'Angers (Maine-et-Loire) du er au III s. apr. J.-C. Aspects de la vie domestique et typologie, sous la direction de Martial Monteil (université de Nantes) et Maxime Mortreau (Inrap). 
Semelin Swann (2021), Production et consommation du verre antique dans la ville portuaire de Rezé/Ratiatum (Loire-Atlantique), sous la direction de Martial Monteil (université de Nantes) et Stéphanie Raux (Inrap).

Tressard Ophélie (2021), Orléans/Cenabum (Loiret) entre la fin de La Tène moyenne et le début du Haut-Empire. Synthèse cartographique via une approche chronofonctionnelle, sous la direction de Martial Monteil (université de Nantes), Julien Courtois et Émilie Roux-Capron (Pôle d'archéologie d'Orléans).

Wardroper Ruby (2021), Le Mur de l'Atlantique et les vestiges immergés dans la baie de Bourgneuf, étude de cas sur lîle de Noirmoutier, sous la direction de Gaëlle Dieulefet (université de Nantes) et Antoine Le Boulaire (Inrap).

\section{Thèses de doctorat}

Bossard Stanislas (2021), Cultes et sanctuaires du centre et de l'ouest de la Gaule lyonnaise. Des antécédents gaulois à la fin des Dieux (It s. av.n. è. - Ves. de n. è.), sous la direction de Martial Monteil (université de Nantes).

Jandon Gabard Véronique (2021), Les meubles « en bois des Isles" un savoir vivre atlantique (XVI-XIX' s.), sous la direction de Hélène Rousteau-Chambon (université de Nantes).

Peter Pauline (2021), Archéologie et Histoire des canaux de navigation en France métropolitaine (II s. av. J.-C. XVI apr. J.-C.), sous la direction de Martial Monteil et Jimmy Mouchard (université de Nantes). 